\title{
Effect of Arginine and Lysine Substituents on Intracellular Localization and Photocytotoxicity of Dipropoxy-Bacteriopurpurinimide
}

\author{
Elizaveta Dyakova ${ }^{1,2^{*}}$, Anastasija Efremenko ${ }^{2,3}$, Petr Ostroverkhov ${ }^{1}$, Michail Grin ${ }^{1}$ and Alexey \\ Feofanov 2,3 \\ 1. Institute of Fine Chemical Technology, Moscow Technological University (MIREA), Moscow, \\ Russia. \\ 2. Shemyakin-Ovchinnikov Institute of Bioorganic Chemistry, Russian Academy of Sciences, Moscow, \\ Russia. \\ 3. Biological Faculty, Lomonosov Moscow State University, Moscow, Russia. \\ * Corresponding author: dyakova.e.d@gmail.com
}

A search for effective ways to treat cancer remains one of the priorities of modern medicine and pharmacology. Photodynamic therapy (PDT) is an actual high-tech and non-invasive type of therapy based on local or systemic application of a photosensitizer (PS), which accumulates in tumors [1]. Under local light irradiation of the corresponding wavelength PS produces reactive oxygen species (ROS), which destruct cancer cells. Currently, PDT is effectively used for the treatment of cancer of internal organs, lungs, skin, neck, head and brain [1-3]. Due to the growing clinical significance of PDT, the development of new effective PS having intensive absorption in a near-infrared region as well as the search for ways of their targeted delivery to cancer cells remain important pharmacological tasks $[4,5]$.

Dipropoxy-bacteriopurpurinimide (DPBPI) is PS with the long-wavelength absorption band at $795 \mathrm{~nm}$ and high ability to photoinduced production of singlet oxygen. Using confocal laser scanning microscopy (CLSM) we found that DPBPI penetrates efficiently within human lung adenocarcinoma cancer cells A549 (Fig.1 I) and causes photoinduced cell death in the micromolar range of concentrations. The predominant intracellular localization of DPBPI in A549 cells is lipid droplets (Fig.1 I). Usage of DPBPI is complicated because of its high hydrophobicity and concomitant troubles with obtaining stable aqueous solutions of DPBPI. Accordingly, derivatives of DPBPI with arginine (Arg-DPBPI) or lysine (Lys-DPBPI) substituents in the D-ring of porphyrin cycle were synthesized by us to obtain more hydrophilic PS.

Absorption and fluorescence spectra of Arg-DPBPI and Lys-DPBPI in water emulsion of $1 \%$ solution of Cremophor EL have the same shape and maxima as the spectra of DPBPI. Using CLSM we found that Arg-DPBPI and Lys-DPBPI penetrate within the A549 cells but possess drastically different intracellular distributions. Arg-DPBPI stains cytoplasm of cells diffusely. A small number of stained granular structures, which are distinct from lipid droplets, is also observed (Fig.1 III). Lys-DPBPI forms predominantly granular distribution, which is different from the distribution in lipid droplets (Fig.1 II). Comparing photoinduced cytotoxicity of PS, we found that Arg-DPBPI and Lys-DPBPI are five- and ten-fold more active than DBBPI, respectively.

In conclusion, arginine and lysine substituents introduced in DBBPI do not affect the absorption and fluorescence properties of a chromophore, change intracellular localization of PS and enhance considerably photoinduced cytotoxicity [6]. 
References:

[1] AM Rkein and DM Ozog, Dermatol Clin. 32(3) (2014), p. 415.

[2] A Kawczyk-Krupka et al., Photodiagnosis Photodyn Ther. 12(3) (2015), p. 545.

[3] MC Fargnoli and K Peris, Future Oncol. 11(22) (2015), p. 2991.

[4] AF Luz et al., Lasers Surg Med. 50 (2018), p. 451.

[5] AV Efremenko et al., Photochem Photobiol Sci. 11(4) (2012), p. 645.

[6] The financial support of RFBR grant 19-04-00854 is acknowledged.
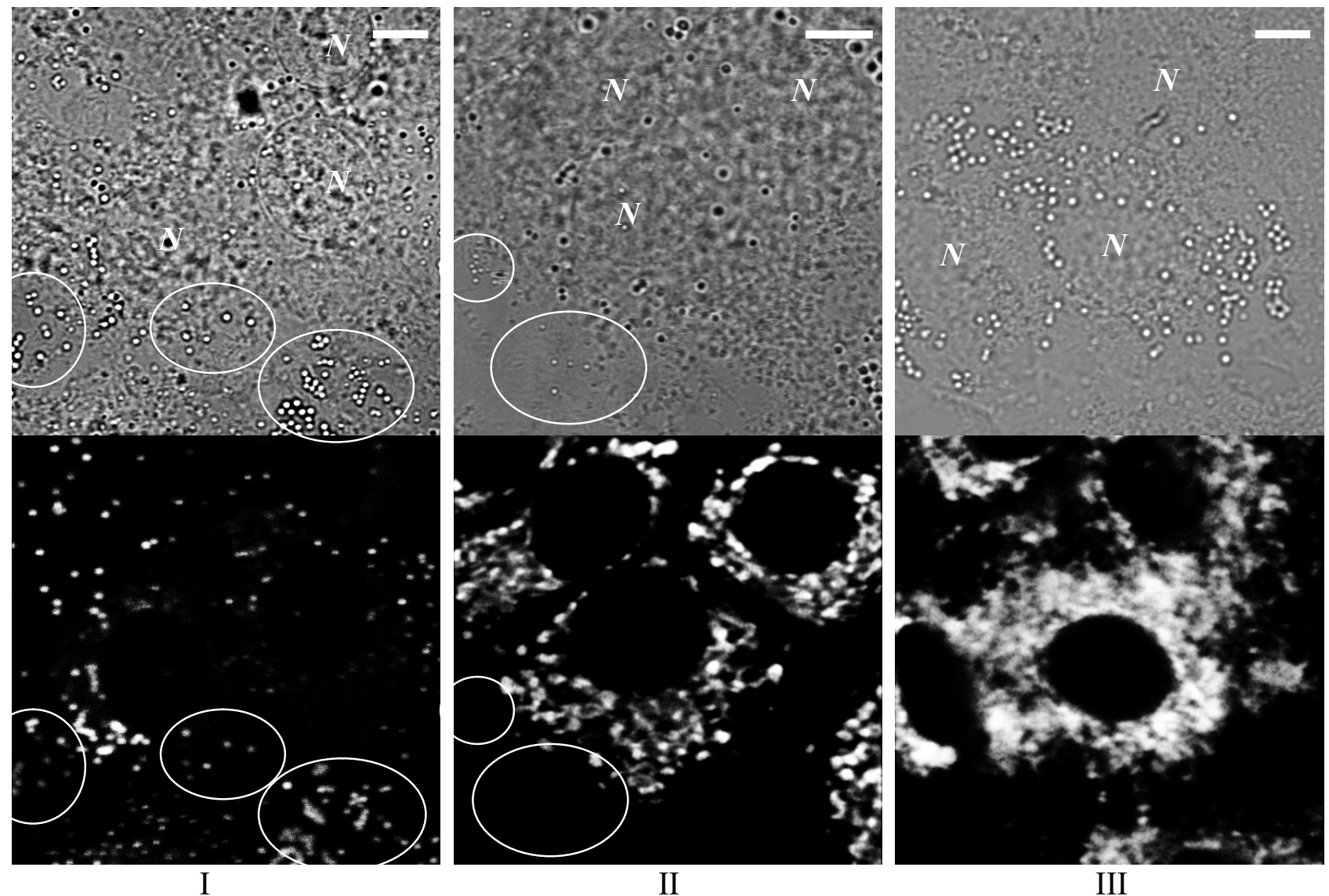

Figure 1. Intracellular distributions of DPBPI (column I), Lys-DPBPI (column II) and Arg-DPBPI (column III) in A549 cells.

Top images - transmitted white-light images of cells. $N$ marks nucleus; bar represents $5 \mu \mathrm{m}$.

Bottom images - confocal fluorescence images describing subcellular distributions of the studied compounds. Cells were incubated with compounds $(2 \mu \mathrm{M})$ for $3 \mathrm{~h}$. Characteristic regions with lipid droplets are marked with ovals in the images. 